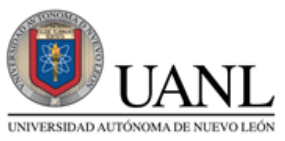

FACPYA
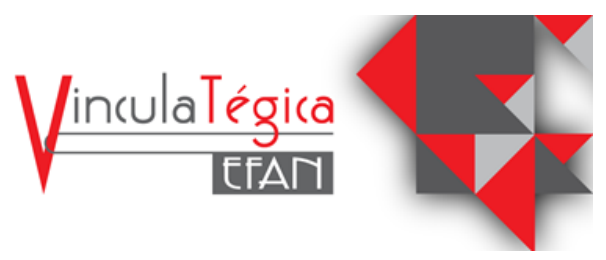

\title{
El impacto de la Responsabilidad Social Empresarial en la crisis COVID 19 en México
}

\author{
Angélica Hernández Leal ${ }^{1}$, Cesar Augusto Hernández González ${ }^{2}$ y Silvia Padilla Loredo ${ }^{3}$ \\ ${ }^{1}$ Universidad autónoma del Estado de México, ahernandezle@uaemx.mx, Av. Bordo de Xochiaca s/n Col. \\ Benito Juárez, 5535215671, \\ ${ }^{2}$ Universidad autónoma del Estado de México,cahernandege@uaemx.mx, Av. Bordo de Xochiaca s/n Col. \\ Benito Juárez 5536807655 \\ ${ }^{3}$ Universidad autónoma del Estado de México, spadillal@uaemx.mx, Av. Bordo de Xochiaca s/n Col. Benito
} Juárez, 5580313502

Información del artículo revisado por pares

Fecha de aceptación: junio-2021

Fecha de publicación en línea: diciembre-2021

DOI: https://doi.org/10.29105/vtga7.2-35

\begin{abstract}
Resumen
El confinamiento derivado de la crisis mundial por la pandemia COVID 19 durante el último año, ha provocado fuertes riesgos de diferente índole, de salud, económica, humanitaria, comercial, y laboral entre otras; la resiliencia empresaria ha sido puesta a prueba de diferentes formas, en sus estrategias administrativas de contingencia, y responsabilidad social (RSE) para sobrevivir a los actuales retos y coadyuvar a detener de alguna manera la debacle económica en todos los niveles. El objetivo de este documento es analizar el impacto de RSE como atemperante para resguardar la vida humana y disminuir de alguna forma la caída mercantil derivada del fenómeno en curso; el método de investigación utilizado es mixto con diseño deductivo, con énfasis en las variables: la situación pandémica como la amenaza a afrontar y la habilidad de las empresas para manejar esta condición a partir del estudio de catorce empresas de diferentes giros y tamaños, observados bajo un encuadre teórico de contingencia y responsabilidad social empresarial que permite entender las interacciones entre las variables presentadas, con resultados que aportan a la comprensión de qué prácticas contribuyen de mejor forma al problema que hemos vivido en los últimos meses
\end{abstract}

\begin{abstract}
The confinement derived from the global crisis due to the COVID 19 pandemic during the last year, has caused strong risks of different kinds, health, economic, humanitarian, commercial, and labor among others; Business resilience has been tested in different ways, in its contingency management strategies, and social responsibility (CSR) to survive the current challenges and help to somehow stop the economic debacle at all levels. The objective of this document is to analyze the impact of CSR as a temper to protect human life and reduce in some way the commercial decline derived from the current phenomenon; The research method used is mixed with emphasis on the variables: the pandemic situation as the threat to be faced and the ability of companies to manage this condition, from the study of fourteen companies of different types and sizes, observed under a theoretical framework of contingency and corporate social responsibility that allows understanding the interactions between the variables presented, with results that contribute to the
\end{abstract}


Palabras clave: Estrategia Responsabilidad social, Pandemia empresarial,

understanding of what practices contribute in a better way to the problem we have experienced in recent months

Keywords: Business strategy, Social responsibility, Pandemic

JEL: M140 Corporate Culture; Diversity; Social Responsibility

\section{INTRODUCCIÓN}

En diciembre del 2019 y hasta la fecha- 15 meses después- el mundo hasta entonces conocido, sufrió una hecatombe de proporciones descomunales a tal grado que hasta la fecha muchos países van cayendo en una debacle económica; esto obedece al problema de salud que se presume inició en China. Casi de forma inmediata la mayoría de fronteras se hallaban cerradas con controles estrictos y por ende la crisis mercantil se vino en cascada; aunque se habla de indicadores macroeconómicos; no son las naciones quienes comercializan bienes, servicios y materia prima, sino las unidades de negocios y los seres humanos según el mercado organizacional o de consumo (Balddwin \& Tomiura, 2020).

$$
\text { La comunidad empresarial }
$$
internacional se ha valido de distintas herramientas para salir avante, la integración del comercio electrónico, las alianzas estratégicas de distintas índoles, pero principalmente ha sido solidaria $y$ responsable hacia la sociedad y hacia sus empleados, hasta donde sus recursos han resistido, ello ha logrado que la economía mundial no haya colapsado completamente y se resista hasta la reactivación. (Daimiel, 2020).

Una de las principales características que ha desarrollado el escenario pandémico actual es la resiliencia universal, es decir, tanto de seres humanos como de organizaciones, esto ha sido imprescindible para aminorar el impacto económico de la crisis sanitaria a nivel mundial; la actitud responsable y solidaria de las empresas hacia la sociedad nunca ha sido más necesaria.

El sector empresarial ha venido enfrentando un constante desafío de supervivencia durante los últimos 15 meses, la crisis pandémica no ha respetado giro, tamaño o ubicación; el riesgo de fenecer se ha esparcido a todo el globo.

Es primordial que el liderazgo empresarial esté focalizado, en escenarios y en anteponer el desarrollo de planes de acción $\mathrm{y}$ tareas que perfeccionen los recursos con los que se cuenta actualmente. Asimismo, el resguardo del capital humano es necesario y preferente.

Para continuar con el rumbo de la 
economía mundial, se ha optado en estos meses por formas alternativas de trabajo, esto se refiere al home office. La definición para el trabajo de oficina en casa requiere tanto de una visión preventiva como de obtención de recursos que faciliten la participación de los colaboradores para mantener y alcanzar el rendimiento requerido en la medida de lo posible.

Salvaguardar a los clientes o consumidores se vuelve más prioritario que nunca, el establecimiento de filtros sanitarios se alinea a las recomendaciones de las autoridades competentes, no sólo es parte del servicio del establecimiento al cliente, se convierte en un tema de ética comercial y responsabilidad social. Por lo anterior, el propósito de este documento es analizar si la responsabilidad social empresarial ha impactado de alguna forma en resguardar la vida humana y disminuir de alguna forma la caída económica derivada del fenómeno en curso.

La referencia teórica, servirá para entender la interacción de las categorías y variables con el fenómeno en cuestión, infiriendo resultados que aporten a entender qué prácticas aportan de mejor manera al problema que hemos vivido en los últimos meses

El enfoque metodológico es deductivo, partimos de un marco referencial que nos permite ubicar ciertas categorías conceptuales en las prácticas aplicadas por las empresas, a continuación mostramos el método utilizado con herramientas mixtas de análisis, finalmente se presentan los resultados del estudio empírico de la investigación, cerrando con las conclusiones.

\section{MARCO TEÓRICO}

Para ubicar las variables de investigación en un contexto situado de conocimientos pandemia, empresa, responsabilidad socialse ha construido un marco teórico que permita entender el enfoque de análisis, así como el estado del arte de autores que escriben sobre el entorno, encontrando apropiada la teoría de la contingencia que relaciona perfectamente dos de las variables, así como la responsabilidad social empresarial, que será un factor prioritario para la supervivencia social y empresarial.

\subsection{Responsabilidad Social Empresarial}

Hablar de Responsabilidad social empresarial, es trasladarse a la década de los 80 's donde cobró fuerza este concepto que ha tenido un intenso recorrido por el mundo ambiental (Samaniego, 2014). Se le ubicaba en un inicio; en el área comercial que se le ha visto como una forma de identidad de los clientes y usuarios (Correa, 2005); en el rubro mercantil, como un factor de desarrollo económico a través de la conducta ética de las empresas hacia los empleados; (Hernández y Jiménez, 2017) 
La implicación "social" de la RSE, tiene tres acepciones: a) como respuesta a los intereses o necesidades de una comunidad; b) cómo las obligaciones contraídas con los grupos de interés; y en su más simple concepto cómo el deber hacia el contrato social - donde existe una obligación mutua de satisfacción- principalmente con empleados, clientes y proveedores.

Responsabilidad moral: que implica la evaluación no solo del efecto sino las causas que lo provocan, (Argangoña, 1998); de esta forma quien ejecuta la acción lo hace muy consciente de que sus actos serán juzgados por las normas sociales y éticas de una sociedad; tanto de forma interna como externa; por lo tanto estará listo para asumir sus actos y sus consecuencias.

La referencia obligada en este indicador de RSE, es el marco normativo de la Ley Federal del Trabajo (D.O.F. 2019) que aglutina el deber ser de las empresas para sus empleados, expresando derechos y obligaciones, al respecto dada la contingencia y necesidad de adecuación por parte de empresas y trabajadores, el pacto de reducción de salario fue una alternativa ante el despido, sin embargo, como se observará, algunas empresas no tuvieron la necesidad de hacerlo y otras incluso tuvieron ganancias.

Responsabilidad causal: relación directa entre las acciones y sus resultados, causando responsabilidad sobre quienes tienen influencia de estos hechos, siendo el ejemplo más clásico, la obligación que tienen los dueños de limpiar la suciedad generada por sus mascotas, la obligación que tienen las empresas y los individuos a subsanar cualquier daño que pudieran provocar con sus actos, entre otros (Volpentesta, 2021); también en este punto tiene cabida la condición económica actual, ya que estando bajo observación por la sociedad entera, las decisiones tomada por los líderes de cada unidad de negocios, tendrá efecto en tres aspectos principales:

Con la reactivación económica local al generar salarios que provocarán consumos a corto plazo,

Con la lealtad de los empleados, al sentirse protegidos por el patrón y obligados a responder con productividad.

Con la fidelidad de sus clientes; al observar acciones solidarias hacia la comunidad entera.

La RSE es parte de un acuerdo tácito, desde las perspectivas social, moral y causal, la empresa forma parte de una comunidad que existe por ella y para ella, con el deber implícito de en su rol de participante, mejorarla . Ferrell y Hirt (2004) destacan la obligación de cada empresa de optimar su efecto positivo, fundamentalmente con conducta ética que impacte en lo económico para sus empleados y socios, generador de 
bienestar - para sus clientes, empleados, socios- reduciendo su efecto nocivo que habitualmente es ambiental, para el mundo en general.

Nunca como en el momento actual ha sido más necesaria la RSE en el ámbito mundial para coadyuvar a detener la debacle no sólo económica sino crisis humanitaria por la pandemia.

\subsection{La teoría de la contingencia}

La teoría de la contingencia, enfatiza la relación entre cualquier organización y sus diferentes entornos; en este caso en especial el macro entorno no controlable en absoluto para la empresa y las decisiones administrativas que si son controlables; la idea central de esta propuesta es que la empresa empleará las acciones administrativas apropiadas acordes a las características de la situación, lejos de las políticas o supuestos establecidos que en su mayoría se enfocan a puntos centrales de problemas macroeconómicos - devaluación, inflación entre otros-, sin contar grandes debacles tales como crisis sanitarias, revueltas sociales, estado fallido entre otras (Donaldson, 2006). En esta investigación se presentan tres escenarios:

Escenario A: las actividades normales de cualquier empresa con alta responsabilidad social.

Escenario B: la reciente crisis pandémica que hasta al corte del 23 de febrero, registró un total de 181 mil 809 muertes por Covid-19 (Secretaría de Salud, 2021), lo que llevó a un cierre empresarial casi generalizado que se ha abierto gradualmente por necesidades y problemas económicos. Las variables exógenas en el entorno suponen un alto grado de adversidad al poner en riesgo la vida, además del efecto económico que conlleva el confinamiento para su protección

Escenario C: la decisión de las empresas en condiciones de alta incertidumbre en relación a la responsabilidad social ante los entornos más inmediatos (clientes, proveedores, y empleados).

En ese trazo, se invita a pensar con Czarniawska que los principios universales dejan de aplicarse por no ser flexibles y por tanto falibles para centrarse en los puntos decisivos de la relación de la organización con el entorno; las respuestas empresariales se modelan a partir de esta interacción y la subsistencia de una organización depende de la capacidad de respuesta ante estas adaptaciones (Czarniawska, 2008); las empresas a nivel mundial han echado mano de diferentes recursos y políticas para salir avante de la pandemia.

A decir de Oña-Sinchiguano (2020), afirma que la Teoría de la contingencia es una buena aliada donde la gestión empresarial puede refugiar sus aspectos más vulnerables a 
través de la prospectiva elaborando un plan de trabajo con distintos escenarios para la organización, considerando principalmente el talento humano. Al hablar de vulnerabilidad, el autor define esta fragilidad como una situación crítica afrontada en lo individual, un conjunto o una comunidad donde se distinguen los siguientes elementos: 1) la existencia de riesgos exógenos cercanos, 2) la cercanía de los mismos, 3) eventualidad de sortearlos, 4) las competencias y capacidades físicas o intelectuales para afrontarlos, 5) Los resultados que se esperan o pueden alcanzar (Oña-Sinchiguano, 2020). De esta forma se ubican dos variables principales: la situación pandémica como la amenaza a afrontar y la habilidad de las empresas para manejar esta condición; la medida en que las organizaciones conozcan y apliquen el manejo de los distintos escenarios, ofrece luces en ubicar qué decisiones las llevan a elegir y construir la mejor opción.

En ese abanico de posibles escenarios y decisiones, se sugiere plantear la contingencia en relación a la teoría de la responsabilidad social empresarial; el sector empresarial ha venido enfrentando un constante desafío de supervivencia durante los últimos 15 meses, la crisis pandémica no ha respetado giro, tamaño o ubicación; el riesgo de fenecer se ha esparcido a todo el globo; en este contexto, es prioritario que los líderes empresariales se centren en la ejecución de planes de acción que le permitan una operación efectiva y sin riesgo para su principal recurso, los seres humanos (Universidad Católica de Colombia, 2020).

Aunque un estudio de Online Carreer Center (OCCMundial); señala la tendencia del Home office como alternativa laboral, las empresas en general no consideran esta modalidad como sinónimo de productividad además de las variantes que implica tales como conexiones, dispositivos, electricidad entre otros, no obstante, su sentido solidario las inclina a hacer uso de ello hasta donde les es posible (2020).

\subsection{La empresa como organización social}

La doble naturaleza exógena y endógena en una organización es resaltada por Hernández y Palafox (2012), en la dualidad del cumplimiento de objetivos, tanto internos, como sociales; en esta dinámica su principal herramienta serán los seres humanos adscritos a cada una de las organizaciones comerciales o de cualquier índole, considerando en el caso de la responsabilidad social empresarial el apoyo a su personal como primera obligación social, como se plasma más adelante en las empresas estudiadas.

De igual forma las empresas se han volcado a ambientes tecnológicos con una doble función, mantener hasta donde les es posible la productividad y guardar las 
observaciones de sana distancia, esto ha provocado el crecimiento exponencial del comercio electrónico que aunque no es una variable específica analizada en el presente documento, si ha facilitado el trabajo desde casa como instrumento de salvaguarda de clientes y empleados (Niño \& Cortés, 2020)

\section{MÉTODO}

\subsection{Planteamiento del problema}

Según datos del INEGI (2020) sobre el Estudio sobre Demografía de los Negocios, se estima que la detrimento económica ha sido el $20.81 \%$ de los 4.9 millones de establecimientos micro, pequeños $\mathrm{y}$ medianos, esto indica un poco más de un millón de unidades de negocios, lo que propició la pérdida de 12 millones de empleos entre abril y diciembre del 2020, derivados de la crisis pandémica (INEGI.2021).

En este contexto se hace necesario revisar los esfuerzos de las empresas por ejercer su responsabilidad social empresarial, apoyando a sus grupos de interés, principalmente a los empleados y clientes; al empleado como principal recurso para la producción de bienes y servicios; a sus clientes, consumidores y/o usuarios para satisfacer sus necesidades y mantener los ingresos empresariales en un círculo virtuoso ventas-ingresos-salarios, con la idea de comprobar que este ejercicio de RSE, no es la causa principal de la desaparición de tan grande número de empresas.

\subsection{Diseño de Investigación}

La presente investigación tiene un enfoque descriptivo, que explica el fenómeno desde la observación no participante, con la intención de evitar cualquier sesgo en la interpretación del fenómeno estudiado; la técnica de investigación es mixta (cuantitativacualitativa), aunque se centra en expresar los resultados de forma estadística de acuerdo a la información encontrada, reconoce la entrevista como la mejor herramienta para la recopilación de información y el posible matiz que ello implica.

Los Ítems considerados en la entrevista abierta con respuestas significativas fueron los siguientes:

Nombre de la empresa,

Giro

¿Qué forma de trabajo han tenido durante la pandemia?

¿Los empleados se han visto afectados en sus percepciones por la crisis pandémica?

¿La empresa ha visto disminuida su cartera de clientes?

¿Han tenido cambios importantes en sus ingresos?

¿Han tenido despidos de empleados?

En la construcción del marco teórico se hace una investigación documental, cuyo 
fin ha sido ubicar los conceptos más adecuados para interpretar los fenómenos ocurridos en las unidades de análisis elegidas. Se han elegido a catorce empresas que operan en México de diferentes tamaños y giros en un muestreo no probabilístico; Otzen y Monterola (2017), indican que la representatividad de una muestra que no obligatoriamente caracteriza al universo, permite la inferencia y la generalización de los resultados observados en ésta.

Este muestreo se utiliza cuando no existe la posibilidad de obtener modelos de forma aleatoria, lo que aplica al presente documento. Las catorce empresas analizadas se obtuvieron a partir de experiencias laborales de personas empleadas en cada una de ellas; esto se identifica como un segmento por conveniencia en respuesta al confinamiento y a la disponibilidad y es recomendada en los casos en que la población es excesiva y no puede ser considerada.

\subsection{Hipótesis}

El supuesto del documento es si la protección de empleos y salarios derivada de la RSE, no es causa de cierre de empresas en esta crisis pandémica del 2020-2021

\subsection{Descripción del método}

Las empresas mexicanas serán abordadas a partir del método empíricoanalítico, adecuado para el análisis del trinomio, pandemia/empresa/responsabilidad social en el control de daños económicosocial, en un entorno de crisis; el instrumento será entrevistas no estructuradas elegidas a partir de la disponibilidad de información en línea con el propósito de no violentar las recomendaciones sanitarias.

Se debe subrayar el uso del enfoque metodológico con el principal objetivo de verificar hasta qué punto puede resistir la responsabilidad social empresarial, una crisis prolongada que a pesar de no haber finalizado, ha cambiado la forma de operar de millones de empresas en México y en el mundo,

\section{RESULTADOS}

En este apartado se analizará las acciones tomadas por cada empresa así como un análisis global en forma gráfica

Total Play, S. A. de C.V. empresa de telecomunicaciones, con un total de 2800 empleados, el $40 \%$ de ellos trabajando como promotores del servicio; con el riesgo concentrado de contagios en pandemia por COVID-19, la empresa de telecomunicaciones impide que los trabajadores, con los sueldos más bajos, se queden en sus casas. Los trabajadores acceden a seguir tocando los timbres de las casas porque la mayoría de sus promotores ganan su sueldo bajo comisión con una 
jornada de trabajo de 9 horas. La empresa aumentó sus ventas durante la pandemia por la necesidad de entretenimiento familiar, y a la implementación de home office que ayudo que Total Play se pudiera fortalecer con un aumento del $39 \%$ sumando 5.6 millones de suscriptores (Gaytan R, 2021).

Autobuses de Oriente, S.A. de C.V(Ado); Un caso similar, el 50\% de sus 26000 empleados, mayormente operarios de autobús, siguen trabajando, y el personal mínimo administrativo, durante casi 9 meses han estado trabajando en home office, solo con algunas reuniones esporádicas, también han evitado que se viaje con aglomeraciones muy grandes, así como se han preferido los viajes nacionales. (Monteón, 2020)

Nutrisa (Grupo Herdez, S.A. de C.V.), Trabajó con sueldo normal, cuando inició la pandemia los primeros 5 meses cerró por completo y estaban pagando con el sueldo mínimo, hasta la fecha tiene reducción de personal con 3 personas máximo por tienda, cerrando más temprano que en su horario habitual, teniendo a sus empleados con sueldo mínimo a causa de que la mayoría de las plazas aún están cerradas y Nutrisa no está trabajando al $100 \%$ de su capacidad y registra pérdidas del 33\%. (Anaya, 2020)

Grupo Coimportex S.A. de C.V; empresa de servicios aduanales, logísticos y legales en temas de comercio exterior. Descansó a sus trabajadores al inicio de la pandemia por una semana, posteriormente los intercalaba, es decir, un día asistía la mitad y otro día los restantes durante 3 meses siempre pagando los sueldos normales, gradualmente han pasado de trabajo en casa a presencial, todavía teniendo al $30 \%$ (la población más vulnerable) en colaboración desde su hogar aún con salario completo, la empresa ha aumentado sus ventas un $18 \%$ (Arellano, 2020)

Grupo Juguetrón, S.A. de C. V. Siguió las ordenes del gobierno y estableció cuarentena a sus empleados. Durante los 2 primeros meses siguió pagando con las nominas ya establecidas. Después del 3er mes empezaron a pagar la mitad de nómina. El sector de los juguetes fue uno de los que se vieron totalmente afectados presentando caídas en sus ventas del $40 \%$ ya que no se consideraba indispensable para mantenerse abierto, este sector se vio obligado a cambiarse al mundo online totalmente, pero no funcionó ya que de acuerdo con lo emitido por la PROFECO esta tienda en específico tenía muchas observaciones con su sitio online, faltaba más información para los clientes ya que era muy confuso haciendo que estos no quisieran o no pudieran comprar. (Flores, 2021)

Hidrosina, S.A. de C.V A inicios de pandemia utilizó trabajo en casa con sueldos y horarios normales y sin despidos. Una vez que se pasó a semáforo naranja regresaron a labores con normalidad, actualmente se vieron beneficiados con la pandemia ya que 
ganaron terreno a PEMEX, de acuerdo con cifras de la Secretaria de Energía, entre enero y junio Hidrosina tuvo presencia en el mercado con crecimiento del $60 \%$. (Santillana, 2020)

Nissan Motor Co., Ltd. Los primeros 15 días sus empleados trabajaron con normalidad. Los siguientes 15 días iniciaron labores en casa con sueldo normal. Una semana después su paga se redujo al $50 \%$ hasta el mes de mayo. En junio iniciaron a despedir empleados. La paga continuó en $50 \%$ hasta que el semáforo se puso en naranja, actualmente el sueldo sigue a la mitad con turnos salteados entre compañeros para evitar contagios, tienen rotación de personal evitando aglomeraciones, no hubo despidos solo reducción de salario. (Villanueva, 2020)

Telstock, S. A. de C. V. Empresa desarrolladora de alta tecnología, desde el comienzo de la pandemia se trabajó home office horarios y salarios normales. Las labores en oficinas iniciaron cuando el semáforo paso a naranja, sin embargo, no fue de forma total, regresaron a oficina el $40 \%$ de los trabajadores rotando los días con el otro 60\%. (Garza, 2020)

Damage Contro, S. A. de C.V, empresa que da servicios de diseño de sistemas de cómputo y servicios relacionados, descansó a todos sus empleados con paga normal, exceptuando a los más jóvenes que eran constantemente monitoreados con pruebas COVID y oxímetros, paulatinamente están regresando a trabajar sin bajas significativas en sus ventas. (Durango, 2020)

Empresa de comercialización de productos del Itzmo, (Central de abastos) cerró por 2 meses, sin embargo, eso no afectó la paga de sus 12 trabajadores, pasado este periodo, siguió laborando con regularidad con todas las medidas de seguridad, regresando la mayoría de sus empleados a laborar de formar normal y con sueldos del $100 \%$ en ningún momento durante la pandemia hicieron reducción, tuvieron un crecimiento del $17 \%$ cuando el semáforo estuvo en naranja y la mayoría de los empleados pudieron regresar a trabajar de forma presencial (Camarillo, 2021).

Tiendas de Abarrotes "Ruiz". Es una microempresa que resultó beneficiada con la pandemia debido a que sus ventas se incrementaron en un $50 \%$ porque los vecinos preferían hacer sus compras de productos de primera necesidad y en ningún momento se vio obligada a cerrar; guardando todas las medidas de higiene indicada para la protección de sus clientes y empleados, incluso contrató personal por ampliación de sus horarios (Ruíz, 2020).

Banco Nacional de México, Citibanamex; Tiene alrededor de 36 mil colaboradores, indicó que con excepción del área de consumo y dependiendo de cada área, entre el 50 y 75 por ciento del personal se 
encuentran laborando desde sus casas, sin reducción de sueldo, el restante se encuentran trabajando en las instalaciones con las medidas sanitarias adecuadas, no se vieron afectadas respecto a perdidas, sólo por los momentos de atención a clientes que hubieron de esperar el doble del tiempo que regularmente esperaban para hacer sus trámites, transferencias, depósitos, pagos, consultas etc. (Pastrana, 2020)

Grupo Bimbo, S. A. de C.V.; Recurrió a la modalidad de trabajo en casa, abriendo vacantes de ejecutivo de soporte técnico para este efecto de forma temporal. Actualmente los repartidores trabajan en su horario normal rolando los días para que sea mínimo el riesgo de contagio, se les da el material necesario para seguir las medidas sanitarias, no hubo reducción de personal y no tuvo pérdidas ya que continúo abasteciendo con regularidad con una creciente en su demanda del 37\%. (Pantaleon, 2020)

Jorbee Ingenieria S.A de C.V; Es una firma mexicana especializada en soluciones en movilidad y gestión de estacionamientos, la empresa se vio afectada en el momento que tuvieron que cerrar las plazas y centros comerciales, aunque continuaron generando ingresos gracias a que los grupos que venden productos de primera necesidad no cerraron en toda la pandemia, tuvieron parado su equipo causando pérdidas del 33\% que se debió al semáforo rojo para los centros comerciales, para los grupos como Walmart, Sams, Bodega Aurrera, Superama trabajan con normalidad solo han acatado las medidas sanitarias pertinentes, gracias a eso la empresa no tuvo que despedir a muchos empleados, pero si pagaron solo la mitad de la nomina; no se han bajado sus ventas más allá del $8 \%$ (Ojeda, 2020)

A continuación, se ha preparado una tabla clasificando a las empresas por su resultado comercial, después de observar su responsabilidad social durante la pandemia:

Tabla 1. Clasificación de empresas, de acuerdo a su estado en la pandemia.

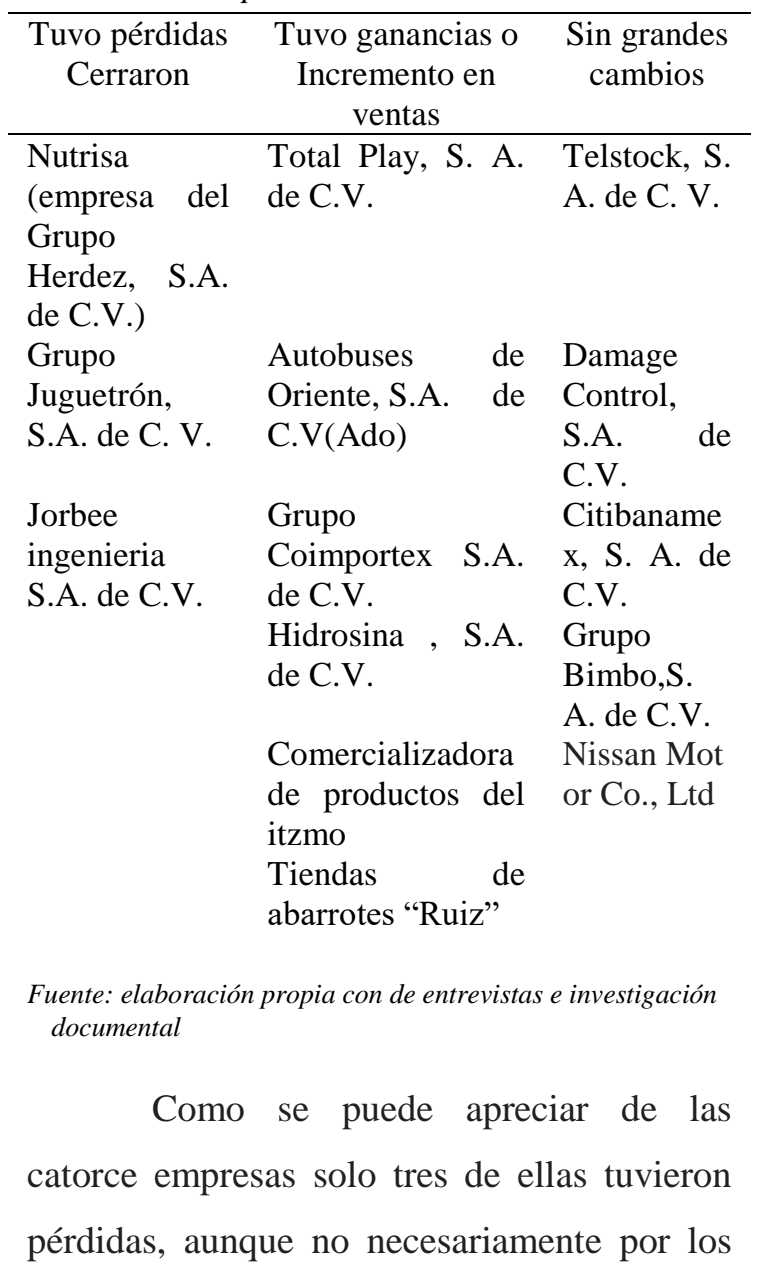


esfuerzos que les significó la RSE en estos tiempos, se presume que el confinamiento de clientes y el entorno de incertidumbre fue un factor determinante para este efecto.

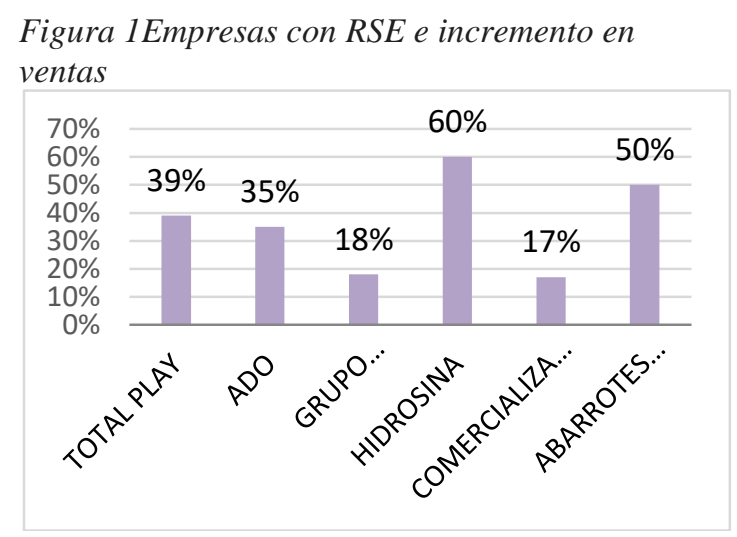

Fuente: elaboración propia con datos de entrevistas e investigación documental

Las cifras de la figura 1 muestran que la RSE realizada por estas empresas, ofreciendo el apoyo a los empleados y clientes, no representó mayor problema en ventas; los empleados al sentirse arropados por la empresa mostraron un mayor compromiso, coadyuvando en buena medida al incremento en ventas dado que la misma ambigüedad laboral podría poner al personal en una difícil situación como fue la de miles de empresas.

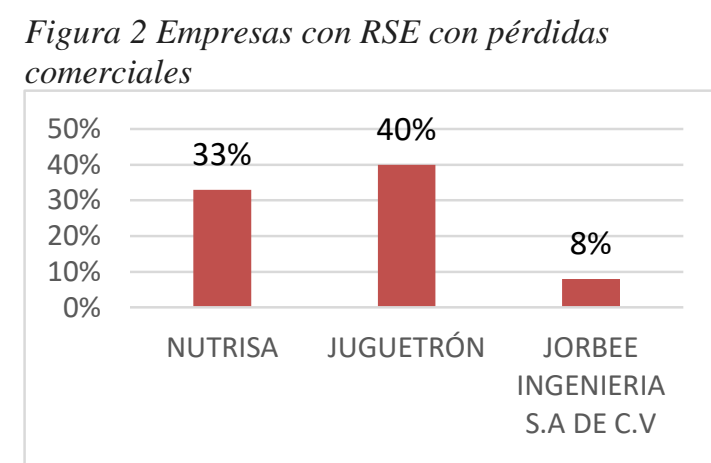

Fuente: elaboración propia con de entrevistas e investigación documental

Por la información recopilada, se pueden inferir múltiples factores que afectaron en las pérdidas y ganancias de las empresas bajo el efecto contingente de la pandemia, así como los mecanismos de adaptación utilizados. A decir de la RSE, su práctica permite explorar algunas reflexiones finales

\section{CONCLUSIONES}

El recorrido a través de los enfoques teóricos de contingencia, responsabilidad social y organización permite observar su vinculación al momento de afrontar la crisis e incertidumbre en la contingencia provocada por COVID19.

En esa tesitura, nos hemos ubicado en el Escenario C; narrar las decisiones de las empresas en condiciones de alta incertidumbre; su relación con la responsabilidad social ha cruzado la esfera exógena (clientes, proveedores, mercado, competencia, etc.) y endógena (empleados, formas de trabajo, prestaciones, salarios, etc).

En el indicador 4 acerca de las contingencias (Competencias y capacidades físicas e intelectuales para afrontar el entorno), nos hemos centrado en la parte fundamental del proceso productivo, los empleados, donde la RSE nos da indicios de 
las decisiones tomadas por la organización y sus efectos.

Así fue posible advertir en su ámbito moral, que las modificaciones a salarios, retiro de prestaciones y despidos, se apegaron sobre todo a las cualidades organizacionales, como la naturaleza del giro comercial que tenían.

Por ejemplo; empresas dedicadas a la tecnología, financieras o comercializadoras; no modificaron su política de responsabilidad social, salarios ni prestaciones. Por el contrario, aquellas que requerían venta directa y en punto como Bimbo y Nutrisa, expusieron en mayor medida a sus empleados, de forma irónica; esa decisión mantuvo la cadena de valor y mantuvo para la primera los salarios y prestaciones.

Otro factor observado en lo causal, fueron las dimensiones de las empresas y su capitalización antes de la pandemia, Juguetrón es un ejemplo que a pesar de intentar sostener la RSE, su dinámica comercial y su falta de recursos, la obligó a despedir personal y hacer ajustes para la sobrevivencia, un acercamiento al ecommerce quizá hubiese traído otras posibilidades.

Además se encontró que aunque el estudio de la OCC (2020) señala que las empresas no encuentran el home office como una estrategia productiva, si ha sido una alternativa aceptable para esta situación en particular que les ha permitido de alguna forma de seguir operando, lo que afirma lo expuesto por Niño y Cortés (2020) quienes indican que los medios virtuales se harán indispensable en esta crisis en dualidad, tanto para ofrecer los bienes y servicios como para hacer posible que se responda a esa oferta, es decir que el trabajo en casa se aplique para cerrar el círculo comercial en este confinamiento.

$$
\text { Por último se resaltan dos }
$$
reflexiones, la primera es destacar la idea de resiliencia empresarial no cómo una capacidad de resistencia, adaptación y recuperación a la contingencia, sino como un llamado a la construcción de redes solidarias entre empresas grandes y chicas, cuya finalidad sea asumir la RSE en lo exógeno y pensar más allá de la ganancia concreta y la utilidad; pensar en la acumulación de capital social a largo plazo; entre más estabilidad mayor certidumbre para todos los actores que integran la sociedad.

La otra idea, es considerar que los lazos endógenos de solidaridad empresarial ejercida a través del concepto de RSE en algunas de las empresas analizadas, no han sido motivo de cierre; esto indica que el bienestar de los empleados en las empresas que lo han ponderado no ha sido factor 
determinante para la crisis económica, sino en todo caso, las bajas ventas derivadas del confinamiento, las dimensiones organizacionales, la naturaleza de su giro y su falta de redes comerciales. 


\section{REFERENCIAS}

\section{Entrevistas}

Anaya, V. (6 de noviembre de 2020). La responsabilidad social empresarial en tiempos de covid . (H. A., Entrevistador)

Arellano, M. (noviembre 17 de 2020). La responsabilidad social empresarial en tiempos de covid . (H. A, Entrevistador)

\section{Artículo científico en línea}

Argandoña, A. (30 de abril de 1998). The stakeholder theory and the common good. Journal of business ethics, 17(9):1093-1102. Obtenido de Journal of Business Ethics 17(9):10931102: https://www.researchgate.net/publication/251166996_The_Stakeholder_Theory_and_the_C ommon_Good

\section{Entrevista}

Camarillo, B. (23 de enero de 2021). La responsabilidad social en tiempos de COVID 19. (Quintero, \& L, Entrevistadores)

Libros impresos

Correa, E. (2005). Responsabilidad social corporativa en América; una visión empresarial;. Chile: CEPAL.

Czarniawska, B. (2008). Organizations as obstacles to organizing. Gothenburg Research Institute, School of business, Economics and Law ate University of Gothenburg, Sweden.

Donaldson, L. (2006). The contingency Theory of Organizational Design; Challenges and opportunities, . New York: Springer Ed.

\section{Entrevista}

Durango, P. (18 de noviembre de 2020). La responsabilidad social en tiempos de COVID 19. (L. Quintero, Entrevistador)

\section{Noticia o publicación en página de internet}

Flores, Z (2021). Reglamento sobre Home Office. 11 de abril del 2021 El Financiero, electrónico https://www.elfinanciero.com.mx/economia/estos-son-los-9-derechos-y-5-obligacionesque-debes-saber-si-haces-home-office-segun-la-stps/

\section{Libro impreso}

Ferrel, O., \& Hirt, G. (2010). Introducción a los negocios en un mundo cambiante. México: Mc Graw Hill.

\section{Entrevista}

Garza, R. (18 de noviembre de 2020). La responsabilidad social empresarial en tiempos de Covid 19. (García. C, Entrevistador)

Gaytan R. (14 de enero de 2021). Responsabilidad social empresarial en tiempos de COVD. (Hernández. A., Entrevistador)

Libros impresos

Hernández, A., \& Jiménez, S. (2017). La Responsabilidad social empresarial como factor de 
internacionalización empresarial. En E. Velazquez, \& M. Cruz, Crítica a la

Responsabilidad social; una perspectiva multidisciplinaria (págs. 115-145). Toluca:

Castellanos.

Hernández, S., \& Palafox, G. (2012). Administración teórica, proceso, áreas funcionales y

estrategias para la competitividad. México: Mcgraw Hill .

\section{Entrevista}

Monteón, A. (18 de diciembre de 2020). La responsabilidad social empresarial en tiempos de Covid . (H. A., Entrevistador)

Informes y reportes gubernamentales, institucionales o empresariales

INEGI (2020) Instituto nacional de estadística, geografía e informática; comunicado de prensa 617/20; Resultados de la segunda edición del ecovid-ie y del estudio sobre la demografía de los negocios 2020, https://inegi.org.mx/contenidos/saladeprensa/boletines/2020/OtrTemEcon/ECOVIDIE_DEMOGNEG.pdf

INEGI (2021) Instituto nacional de estadística geografía e informática; comunicado de prensa 115/21 Resultados de la encuesta nacional de ocupación y empleo. Nueva edición (ENOEN) cifras durante el cuarto trimestre de 2020; en https://www.inegi.org.mx/contenidos/saladeprensa/boletines/2021/enoe_ie/enoe_ie2021_02 .pdf

\section{Revista científica en línea}

Niño, B. T., \& Cortés, M. (2020). Comunicación estratégica y responsabilidad social empresarial, escenarios y potencialidades en creación de capital social. Prisma social: Revista de investigación social, 127-158.OCC Mundial . (2020). Termometro Laboral . México: OCC México.

\section{Entrevista}

Ojeda, P. (25 de noviembre de 2020). La responsabilidd social empresarial en tiempos de COVID.

(García. C, Entrevistador)

\section{Revista científica en línea}

Oña-Sinchiguano, B. (2020). La Teoría de la contingencia y la Teoría de Costes en el manejo de la Pandemia en el Ecuador. Koinonia (Fundación Koinoniana), Vol. V No. 4 2542-3088. http://dx.doi.org/10.35381/r.k.v5i4.1004

Otzen, T., \& Manterola, C. (2017). Técnicas de muestreo sobre una población a estudio . Int. J. Morphol, 35(1):227-232,. https://scielo.conicyt.cl/pdf/ijmorphol/v35n1/art37.pdf

\section{Noticia o publicación en página de internet}

Pantaleón, I. (2020). El reto de las empresas durante la Pandemia.10 de abril del 2020 Forbes, https://www.forbes.com.mx/negocios-coronavirus-retos-latam-pandemia/

\section{Entrevistas}


Pastrana, W. (22 de noviembre de 2020). La responsabilidad social en tiempos de COVID 19. (García. C, Entrevistador)

Ruíz, P. (22 de septiembre de 2020). La responsabilidad social empresarial en tiempos de COVID.

(García. C, Entrevistador)

\section{Revista impresa}

Samaniego, N. (2014). El Capital en el silo XXI Thomas Piquette. Economia UNAM, 125-129.

\section{Entrevista}

Santillana, G. (13 de octubre de 2020). La responsabilidad social empresarial en tiempos de COVID. (García, C Entrevistador)

\section{Conferencia de prensa}

Secretaría de Salud. (2021). Actualización COVID. México: Conferencia de prensa televisada abril 23 del 2021Secretaría de Salud.

\section{Memoria de seminario en línea}

Universidad Católica de Colombia. (2020). Seminario, Causas y efectos, económicos y comerciales de la pandemia covid 19. Bogotá, Cundinamarca, Colombia: Facultad de Ciencias Económicas y Administrativas. En

https://repository.ucatolica.edu.co/bitstream/10983/24667/1/CAUSAS\%2CEFECTOS\%20 Y\%20OPORTUNIDADES\%20SOCIOECON\%C3\%93MICAS\%20DERIVADAS\%20DEL $\%$ 20CORONAVIRUS\%20COVID\%2019.pdf

\section{Entrevista}

Villanueva, J. (18 de noviembre de 2020). La responsabilidad social empresarial en tiempos de COVID. (Quintero, L, Entrevistador)

\section{Revista en línea}

Volpentesta, J. (mayo 4 2021). Confluencia de teorías en torno a la Responsabilidad Social Empresaria (RSE). Obtenido de Ciencias Administrativas, Revista digital FCE-UNLP: https://revistas.unlp.edu.ar/CADM/article/view/2499 